\title{
A DFT Study Toward the Reaction Mechanisms of TNT With Hydroxyl Radicals for Advanced Oxidation Processes
}

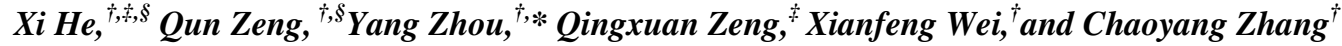 \\ ${ }^{\dagger}$ Institute of Chemical Materials, Chinese Academy of Engineering and Physics, Mianyang 621010 China. \\ ${ }^{\ddagger}$ School of Mechano-electronic Engineering, Beijing Institute of Technology, Beijing 100081, China.
}

\section{Supporting Information}

Table S1: Calculated Reaction $(\boldsymbol{A E})$ and Activation $(\boldsymbol{\Delta G})$ Gibbs Free Energies (298.3K) for Main Reactions in Four Pathways (kcal/mol)

\begin{tabular}{|c|c|c|c|c|c|c|}
\hline Reactions & $\Delta E^{a}$ & $\Delta E^{b}$ & $\Delta G^{a}$ & $\Delta G^{b}$ & $\Delta E^{a, c}$ & $\Delta G^{a, c}$ \\
\hline $\mathrm{TNT} \rightarrow \mathrm{IN} 1 \mathrm{R}$ & 2.3 & 4.6 & 10.9 & 5.4 & 10.0 & 12.2 \\
\hline $\mathrm{I} \rightarrow \mathrm{IN} 2 \mathrm{R}$ & 0.1 & 1.2 & 8.4 & 2.2 & 5.8 & 7.9 \\
\hline $\mathrm{I} \rightarrow \mathrm{IN} 2^{\prime} \mathrm{R}$ & 1.6 & 5.1 & 10.1 & 7.3 & 10.3 & 12.7 \\
\hline $\mathrm{IN} 3 \rightarrow \mathrm{II}$ & 43.6 & 41.2 & 43.7 & 43.9 & 41.9 & 45.4 \\
\hline IN3' $\rightarrow$ II & 55.0 & 55.8 & 54.9 & 62.2 & 52.5 & 56.7 \\
\hline $\mathrm{II} \rightarrow \mathrm{IN} 4 \mathrm{R}$ & 0.8 & 2.4 & 7.3 & 3.3 & 6.0 & 7.6 \\
\hline IN6' $\rightarrow$ IV $^{\prime}$ & 77.0 & 77.1 & 76.2 & 83.8 & 71.3 & 74.4 \\
\hline $\mathrm{IN6} \rightarrow \mathrm{V}$ & 71.1 & 69.1 & 70.7 & 71.4 & 64.1 & 66.7 \\
\hline IN7.2 $\rightarrow$ VI. 2 & 58.6 & 58.4 & 58.3 & 64.9 & 41.9 & 44.7 \\
\hline IN7.4 $\rightarrow$ VI. 4 & 63.7 & 62.6 & 63.8 & 68.0 & 47.8 & 50.3 \\
\hline VI. $2 \rightarrow \mathrm{IN} 8.2 \mathrm{R}$ & 0.5 & 1.8 & 8.7 & 1.6 & 8.3 & 10.3 \\
\hline VI.4 $\rightarrow$ IN8.4R & 1.6 & 1.8 & 9.8 & 1.6 & 9.2 & 11.3 \\
\hline $\mathrm{IN9.4} \rightarrow \mathrm{IN} 10.4 \mathrm{R}$ & 0.1 & 1.4 & 8.3 & 1.4 & 6.1 & 8.0 \\
\hline IN9.4 $\rightarrow$ IN10.4'R & 1.0 & 1.1 & 9.6 & 6.9 & 9.5 & 11.8 \\
\hline IN11.4 $\rightarrow$ VII & 83.2 & 79.3 & 73.0 & 43.7 & 113.0 & 117.7 \\
\hline IN11.4' $\rightarrow$ VII & 57.7 & 58.8 & 57.7 & 64.8 & 51.1 & 55.4 \\
\hline
\end{tabular}

